\title{
The Study of seawater COD analyzer based on PC104 bus embedded technology
}

\author{
Hechao Zang ${ }^{1,2, a}$, Junli Liü, 2, b and Zhili Hua ${ }^{1,2, c}$ \\ ${ }^{1}$ Institute of Oceanographic Instrumentation of Shandong Academy of Sciences, Shandong 266100, \\ China; \\ ${ }^{2}$ Shandong Provincial Key Laboratory of Ocean Environment Monitoring Technology, Shandong \\ 266100, China. \\ azanghechao@126.com, b63033234@qq.com, c 455272587@qq.com
}

Keywords: COD, PC104 bus, Analyzer.

\begin{abstract}
This paper discuss the using of the current PC104 bus technology and chemical oxygen demand (COD) on-site analysis technology to do the secondary development on the the seawater chemical oxygen demand analyzer (COD analyzer). The control system of the COD analyzer is formed by PC104 modules.Each PC104 module is used for some specific function,such as AD convering and unit controling.After determining the specific type of the modules, it will install and equip them with all of the external electrical control units including photoelectric signal collector, ozone generator, various valves,fluid pumps and water sample heating devices.System need to complete the electrical connection between the PC104 modules with the PC104 bus. and then install the operating system software to PC104 CPU module which system programming is developed under the debugging $\mathrm{C}++$ compiler.
\end{abstract}

\section{Introduction}

Seawater chemical oxygen demand ${ }^{[1,2]}$ (COD) is an important indicator to characterize the degree of sea water pollution. COD analyzer is developed based on the principle of light-emitting of ozone oxidation in water, Controling the system of COD analyzer needs to have the basic electrical control functions, and also has the ability to handle complex graphical analysis algorithm.It is hard to develop it with traditional embedded microcontroller technology because of technically difficult in achievement. We developed the new COD analyzer by embedded motherboard PCM-3362 based on PC / 104 bus standard, it can do PC / 104 bus communication with dedicated analog I / O modules PCM3180HG and digital I / O module PCM3730 which install as a peripheral circuit,form the whole processing of collecting outside signal and make decision to control all of the units.

Executing unit PCM-3362 as an signal analysis, could collected multiple analog signals of simulate I / O modules PCM3180HG in real time, make external individual electric unit control through the digital I / O module PCM3730 and self-designed dedicated control circuit.

\section{Method of The Study}

\subsection{COD Analyzer System Structure.}

The overall structure of the water COD automatic analyzer is formed by the ozone generator, photoelectric signal conversion systems, optical reaction chamber and PCM-3362 core data processing system,see Figure 1. 


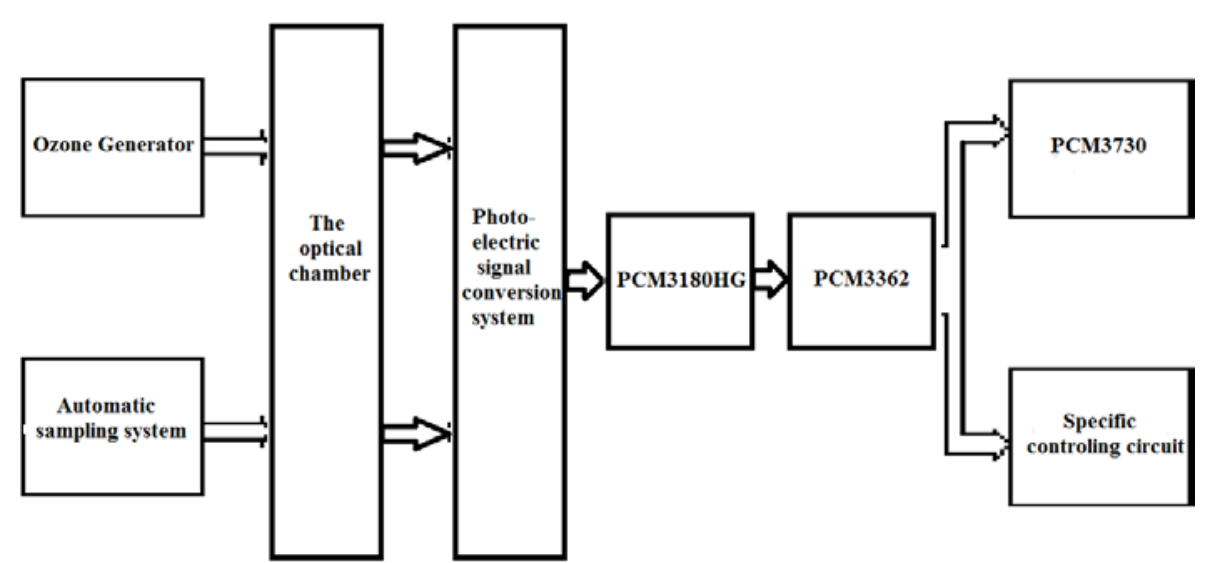

Fig. 1 Seawater COD analytical measurement system structure

The entire measurement process is divided into the following steps: First, the automatic sampling system $^{[3,4]}$ in accordance with program instructions control the injection pump, and sea water samples continuously and steadily are pumped into the reaction chamber. Then, the drying air is sucked into a filter through high voltage and generate ozone which is required for the reaction to produce, the ozone and the seawater sample are pumped into the reaction chamber and mixed together,the oxidation reaction happens,and emit chemical shimmer which is pickuped by the photoelectric signal conversion system, amplified by a micro-computer and send to data processing system for data analysis and fitting graphics algorithms, eventually reduced to give the COD value of seawater.

When the oxidation reaction ${ }^{[5,6]}$ occurs in the optical chamber of the COD analyzer, the chemiluminescence signal collected by the photomultiplier tube is sent to PCM3180HG board which is responsible for signal processing and collection, it provide eight 12 a / D conversion tunnels, the sampling frequency is more than $100 \mathrm{kHZ}$ to ensure timely collection signal of oxidation reaction in the optical chamber.

By the PC / 104 bus, optical signal and the temperature signal collected is converted to 12-bit digital signal and transferred to PCM-3362 motherboard to deal with in real-time.

\subsection{System software development.}

We chose Borland c ++ Builder as the system software development environment,see Figure 2.It is easy to use control group of the $\mathrm{c}++$ Builder to make software development and system interface functions development, when the system needs to communicate to external devices include analog I / O modules PCM3180HG and digital I / O module PCM3730 through PC / 104 bus, we use the device driver function provided by Advantech through learning demonstrations to control these two external devices. Advantech Device Manager as Driver software could be installed in Windows systems when using the device driver function, then add "\#include C: \Program Files $\backslash$ Advantech $\backslash$ adsapi $\backslash$ Include $\backslash$ Driver.h" when the main program is initialized, so that you can call device driver functions in the the driver function library during development.

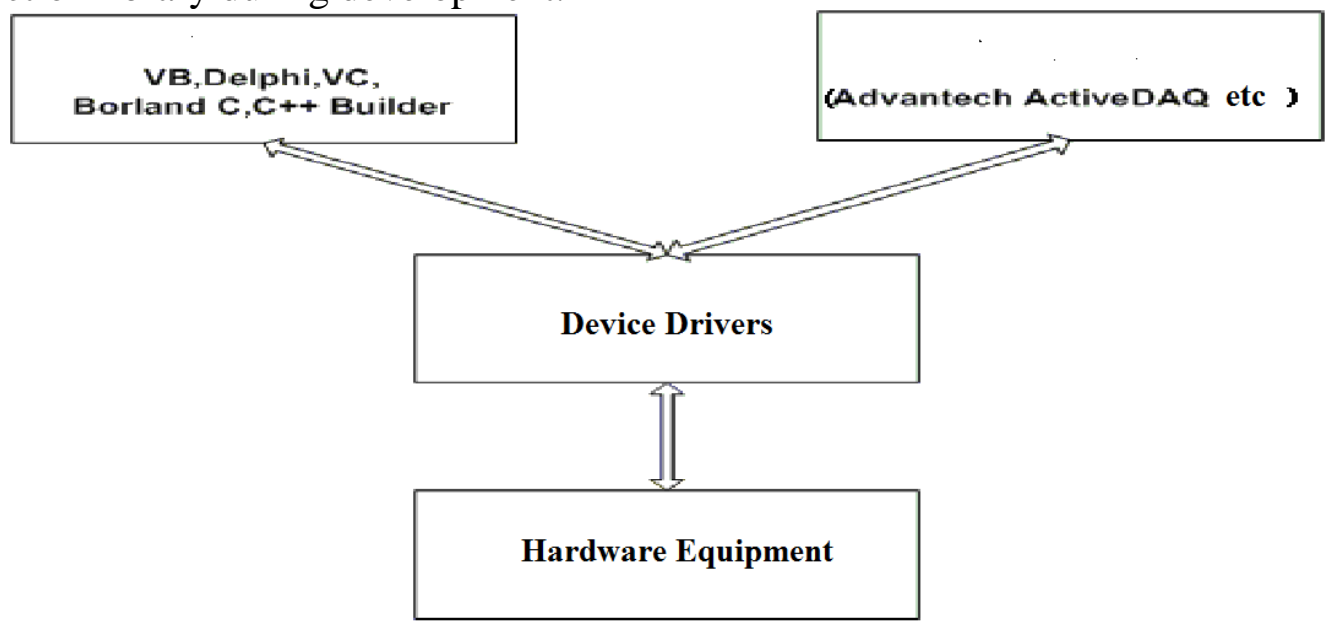

Fig. 2 Software Development Environment Chart 
In this development environment ${ }^{[7]}$, we compile the object-oriented programming is available in the Windows operating system. When we use these driver functions to write system programs designed performance functions for different interface objects, and these functions calls and integrates the function of the device driver.Through varying the parameter variable of the function,the specific device port could be controlled, the following is the main interface for digital I / O module PCM3730 to make function calls.

// Set the port to send commands

bool Tmain::SetPortCmd(unsigned char DataLatch)

\{

short nOutEntries;

long dwDeviceNum;

ErrCde = DRV_DeviceGetList(\&DeviceList[0], MaxEntries, \&nOutEntries);

if (ErrCde !=0)

\{

DRV_GetErrorMessage(ErrCde, szErrMsg);

Application->MessageBox(szErrMsg, "Error!!", MB_OK);

return false;

\}

dwDeviceNum $=$ DeviceList $[0] . d w D e v i c e N u m ;$

ErrCde = DRV_DeviceOpen(dwDeviceNum, \&DeviceHandle);

if (ErrCde $!=0)$

\{

DRV_GetErrorMessage(ErrCde, szErrMsg);

Application->MessageBox(szErrMsg, "Error!!", MB_OK);

return false;

\}

ptDioWritePortByte.port $=0$;

ptDioWritePortByte.mask = 0xff;

ptDioGetCurrentDOByte.port $=0$;

ptDioGetCurrentDOByte.value $=$ \&uDoReadValue;

if $(($ ErrCde $=$ DRV_DioGetCurrentDOByte(DeviceHandle,

(LPT_DioGetCurrentDOByte)\&ptDioGetCurrentDOByte)) != SUCCESS)

\{

DRV_GetErrorMessage(ErrCde,(LPSTR)szErrMsg);

Application->MessageBoxA((char* )szErrMsg, "Driver Message"); return false;

\}

ptDioWritePortByte.state $=$ DataLatch;

if $(($ ErrCde $=$ DRV_DioWritePortByte(DeviceHandle,

(LPT_DioWritePortByte)\&ptDioWritePortByte)) != SUCCESS)

\{

DRV_GetErrorMessage(ErrCde,(LPSTR)szErrMsg);

Application->MessageBoxA((char *)szErrMsg,"Driver Message");

return false;

\}

//Waiting for confirmation

return(true); 
\}

We just need to change the value of the parameter variable DataLatch, and we can control the opening and closing of the digital I / O points in the program's main interface, it is very convenient to use them.

\section{The Algorithm of Graphical analysis}

System software includes a graphical analysis algorithm to characterize the chemistry of seawater COD optical signal.After the initial setup of the algorithm is completed, the software can restore the strength of the case and the graphic description of chemical optical signals throughout the measurement process, obtained the COD value of seawater by fitting algorithm. We compared the national standard method COD value and the fitted value of seawater samples, the results shown in Figure 3 below. Fitting between COD value that COD analyzer measured and national standard method COD value ,the correlation coefficient $\mathrm{R}=0.97$, both direct measurements show good linear relationship, see Figure 3.
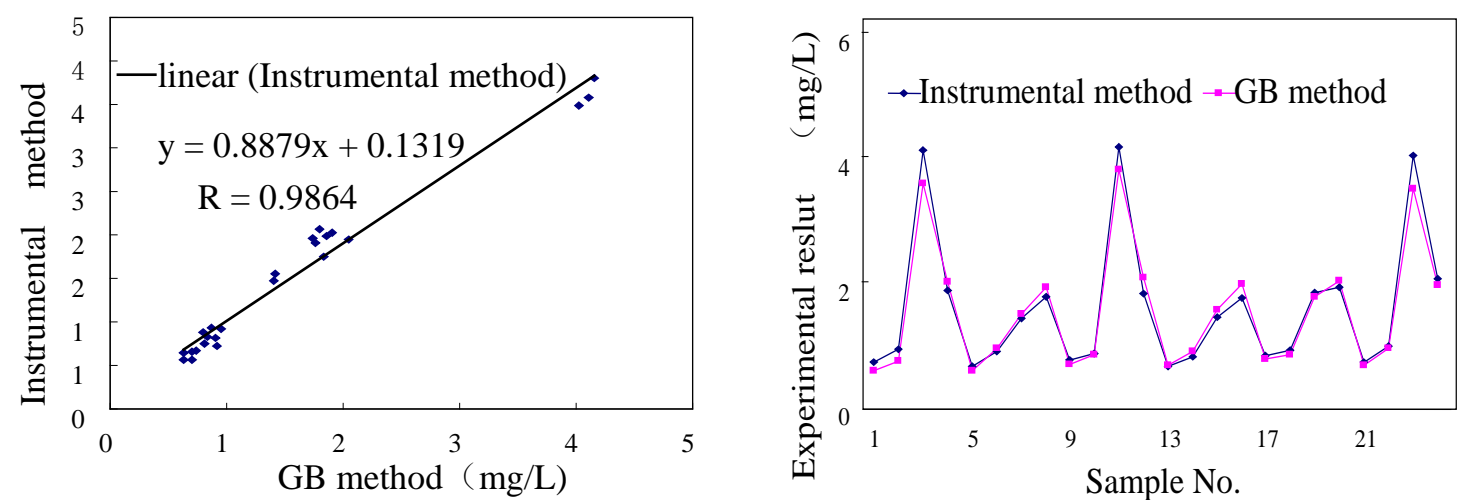

Fig. 3 Comparing the average values of instrumental method and GB method experimental data Linear relation analyzed (left) Average value comparison (right)

\section{Conclusion}

The COD analyzer performs well in these aspects, stably running, highly automation, easy operation and fast measurement (5 minutes a sample), no warm-up, no need chemical reagents and other advantages; The linear relation of instrumental method and GB method results is good, correlation coefficient $\mathrm{R}=0.97$, the results of these two methods show certain coherency; The COD analyzer performs good precision, low detection limit, after further improvement, it can be gradually used for the analysis of seawater samples.

\section{Acknowledgment}

The project is supported by the International Science\&Technology Cooperation Program Of China (2014DFR60490).

\section{References}

[1]. CM O'Brien, CJ Fox, B Planque, J Casey, et al. Climate variability and North Sea cod. Nature. Vol. 404(2000) No. 6774, p. 142-142.

[2]. GA Rose, B Deyoung, DW Kulka, SV Goddard, GL Fletcher, et al. Distribution shifts and overfishing the northern cod (Gadus morhua): a view from the ocean. Canadian Journal of Fisheries \& Aquatic Sciences. Vol. 57(2011) No. 3, p. 644-663. 
[3]. A Engås, S Løkkeborg, E Ona, AV Soldal, et al. Effects of seismic shooting on local abundance and catch rates of cod ((Gadus morhua) and haddock )(Melanogrammus aeglefinus). Canadian Journal of Fisheries \& Aquatic Sciences. Vol. 53(2011) No. 10, p. 2238-2249.

[4]. RA Myers, NG Cadigan, et al. Was an increase in natural mortality responsible for the collapse off northern cod. Canadian Journal of Fisheries \& Aquatic Sciences. Vol. 52(2011) No. 6, p. 1274-1285.

[5]. I Mantzouni, H Sørensen, RB O'Hara, BR Mackenzie, et al. Hierarchical modelling of temperature and habitat size effects on population dynamics of North Atlantic cod. Ices Journal of Marine Science. Vol. 67(2010) No. 5, p. 833-855.

[6]. P.S. Epstein, M.S.Pleasst, et al. On the stability of gas bubbles in liquid-gas solution. Chem.Phys. Vol. 18(1950) , p. 1505-1509.

[7]. L.Somoza, V. Diaz-del-Riob, R. Leon, et al. Seabed morphology and hydrocarbon seepage in the Gulf of Cadiz mud volcano area. Marine Geology. Vol. 195(2003) , p. 153-176. 